\title{
Tidal breathing parameters in the first week of life and subsequent cough and wheeze
}

\author{
B Yuksel, A Greenough, F Giffin, K H Nicolaides
}

\begin{abstract}
Background - Assessment of tidal breathing parameters may be a useful method of predicting respiratory problems in early childhood. Low values of TPTEF/TE (the ratio of the proportion of time to reach peak tidal expiratory flow to total expiratory time) outside the neonatal period have been significantly related to respiratory tract illness with wheezing in boys in the first year of life.
\end{abstract}

Methods - TPTEF/TE measurements in the perinatal period were evaluated in nonsedated infants and the predictive value of this early measurement for subsequent respiratory morbidity during infancy was assessed. Flow during tidal breathing was measured while the infant slept quietly in a plethysmograph using a Fleisch pneumotachograph inserted into an infant face mask. Recruitment continued until traces from 60 infants with 10 consecutive flow curves without artefacts were obtained. In addition, plethysmographic measurements of airway resistance (Raw) and thoracic gas volume (TGV) were measured and specific conductance (sGaw) calculated. Parents recorded their infant's cough and wheeze during the first 12 months of life.

Results - Sixty five measurements were made in 60 infants with a mean age of two days and gestational age of $\mathbf{4 0}$ weeks. Two observers separately calculated TPTEF/TE ratios on 25 traces randomly selected from the pool of 60 . The mean difference between the two observers was -0.004 (limits of agreement 0.048 to $-\mathbf{0 . 0 5 6}$ ). Thirteen infants became symptomatic (wheeze, with or without cough); their median TPTEF/TE ratio (0.349) was significantly lower than the rest of the cohort (median 0.412) and they also had significantly higher Raw and lower sGaw. The positive predictive value of a low TPTEF/TE ratio, however, was only $41 \%$.

Conclusions - These results suggest that the use of this test in the prediction of future respiratory disease in an individual is limited.

(Thorax 1996;51:815-818)

Keywords: infancy, tidal breathing parameters.

Respiratory problems are common during infancy. Accurate predictive tools are therefore desirable so that intervention strategies can be applied appropriately. The results of airway resistance (Raw) measurements are useful but may require the patient to be sedated. ${ }^{1} \mathrm{~A}$ technique to measure tidal breathing parameters (the proportion of time to reach peak tidal expiratory flow to total expiratory time (TPTEF/TE ratio)), previously used in adults, ${ }^{2}$ has recently been employed in infants, some of whom were not sedated. Martinez et $a l^{\beta}$ reported that boys who later developed lower respiratory tract illness with wheezing had significantly lower values of TPTEF/TE at the mean age of approximately two months. Assessment of lung function at that age, however, necessitates recalling infants to hospital and is thus difficult to apply universally. We felt it important to assess whether measurement of TPTEF/TE in the first week of life, when infants are still in hospital with their mothers, provided a useful prediction of subsequent respiratory morbidity and whether such a relationship, as previously described, ${ }^{3}$ was dependent upon sex.

\section{Methods}

Infants were eligible for entry into the study if they had no congenital anomalies, were born at term, and had no respiratory problems in the immediate perinatal period. Infants who fulfilled the eligibility criteria were recruited from an ongoing study examining the effects of first trimester antenatal investigations which were being undertaken because of maternal age or parental anxiety. In that study lung function measurements were made in the first week of life and the infants were subsequently followed until at least one year of age. The parents kept diary cards on which the presence of respiratory symptoms (wheeze and cough) were recorded. They were contacted on two separate occasions and the infant's symptoms were retrospectively reviewed from birth to the first contact and between the first and second contact, respectively. The follow up study was approved by the King's College Hospital ethics committee and parents gave informed consent before entry into the study.

Infants were seen in the Paediatric Respiratory Laboratory. Lung function measurements were made 30-60 minutes after a feed during quiet sleep without sedation. The infants were studied in the supine position lying in a whole body plethysmograph. Tidal breathing parameters were measured before estimation of thoracic gas volume and airway resistance. Flow during tidal breathing was measured while the neonate breathed through an infant 
face mask. The mask was connected to a rebreathing bag via a heated Fleisch pneumotachograph and was sealed around the infant's nose and mouth using silicone putty to ensure an airtight seal. The dead space of the equipment was $15 \mathrm{ml}$. The flow signal was calibrated with a rotameter and was linear between zero and $121 / \mathrm{min}$. Flow and volume signals were collected on ultraviolet sensitive paper. The shape of the tidal breathing expiratory curve was studied. The time to reach peak (maximum) tidal expiratory flow (TPTEF/TE) as a proportion of total expiratory time (TE) was measured. The mean TPTEF/TE ratio was calculated from the first 10 regular breaths. Recruitment continued until 60 suitable traces - that is, with 10 consecutive flow curves without artefacts - were obtained.

Thoracic gas volume (TGV) and airway resistance (Raw) were measured using a whole body plethysmograph (Hammersmith Hospital, Department of Medical Engineering, London, UK) with a total volume of 90 litres. The infant breathed through a face mask which was connected to the rebreathing bag via a heated pneumotachograph. The face mask was sealed around the infant's nose and mouth using silicone putty to ensure an airtight seal. The infant breathed through a heated, humidified rebreathing system to avoid box pressure changes due to heating and cooling of respired gas. TGV was measured at the end of normal inspiration and Raw at two thirds of maximum inspiratory flow using the techniques of Dubois et al suitably modified for infants. ${ }^{45}$ TGV was calculated from five breaths during occlusion and the reported value was adjusted to end expiratory lung volume; five separate occlusions were made. Raw was calculated from 10 breaths. Measurements were corrected for the apparatus dead space which included the face mask $(15 \mathrm{ml})$ and its resistance $(8 \mathrm{~cm}$ $\mathrm{H}_{2} \mathrm{O} / \mathrm{l} / \mathrm{s}$ ) measured at flows of between 5 and $15 \mathrm{l} / \mathrm{min}$. The calibration of the plethysmograph was performed as described previously. ${ }^{6}$

An observer who was unaware of the results of the TPTEF/TE measurements evaluated the diary cards. Infants were defined as symptomatic if they wheezed, with or without cough, for more than one day following a respiratory tract infection or wheezed for at least 24 hours without any respiratory tract infection.

\section{STATISTICAL ANALYSIS}

To determine the interobserver repeatability of the measurements, 25 traces were randomly selected and the mean TPTEF/TE ratio for each trace was calculated separately by two observers. The coefficient of variation (CV) was determined for each trace by each observer. The coefficient of repeatability between the two observers' results was calculated according to the method of Bland and Altman. ${ }^{7}$ Differences between groups were assessed for statistical significance using the Mann-Whitney $U$ test. The sensitivity, specificity, and positive predictive value for the development of respiratory symptoms in the first year of life of the median TPTEF/TE of the study population were calculated.

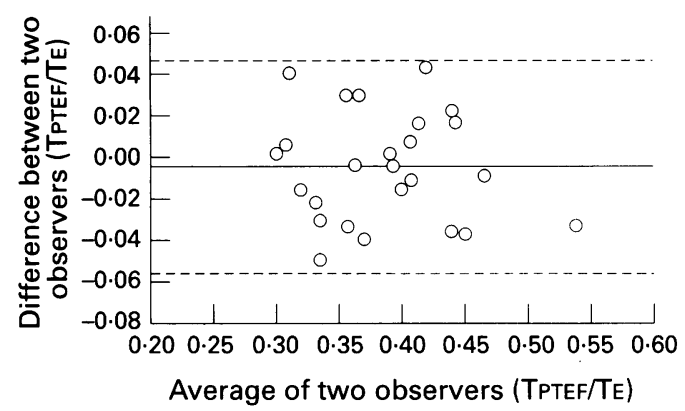

Figure 1 Differences between the two observers in the $T P T E F / T E$ ratio of an individual plotted against the mean TPTEF/TE. The mean difference and $95 \%$ confidence intervals of the mean are demonstrated (solid and broken lines, respectively).

\section{Results}

Lung function measurements were carried out in 65 neonates, but in five cases the traces were unsuitable for analysis. Results were used from the remaining 60 neonates ( 25 boys) who were born at a median gestational age of 40 weeks (range 37-42) and had a birth weight of $3400 \mathrm{~g}$ (range 1800-4960). The median age at the time of investigation was two days (range 1-12). The ethnic origin of the neonates was Caucasian in 42 cases, Afro-Caribbean in 12, Asian in one, and mixed in five. The mean (SD) $\mathrm{TPTEF} / \mathrm{TE}$ values of the two observers were $0.383(0.058)$ and $0.387(0.06)$, respectively, and the mean (SD) difference between paired measurements was $-0.004(0.026)$. The mean (SD) coefficient of variation was 20.4 (8.8)\% (range 3-41) for observer 1 and 15.3 (6.6)\% (range 6-31) for observer 2 . The coefficient of repeatability of the TPTEF/TE results was 0.052 with limits of agreement of 0.048 to -0.056 (fig 1).

Thirteen infants (four boys) were symptomatic at follow up (fig 2). They did not differ from the other 47 with respect to their birth weight, the proportion who were growth retarded, their postnatal age, mode of delivery, ethnic origin, family history of atopy, or respiratory rate (table 1 ). The median TPTEF/TE of the symptomatic group, however, was significantly different from that of the nonsymptomatic infants, as was their airway resistance and specific conductance but not their thoracic gas volume $(p<0.01)$ (table 1$)$. In the total group of 60 neonates there was no significant difference between the median TPTEF/TE of the boys (0.400, range $0.298-0.477)$ and the

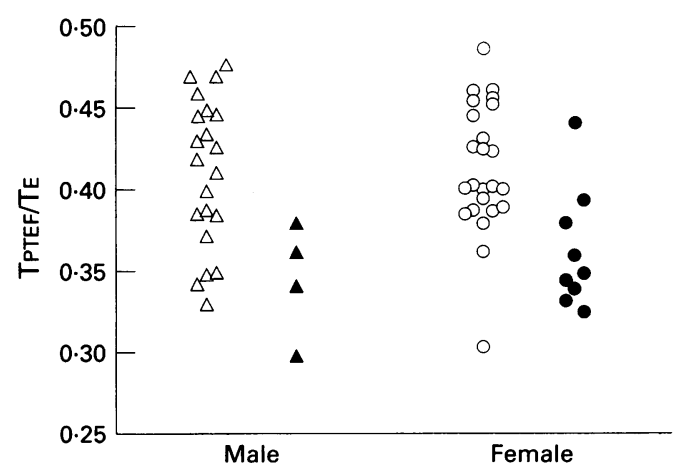

Figure 2 Individual TPTEF/TE results for boys ( $\triangle=$ non-symptomatic, $\mathbf{\Delta}=$ symptomatic) and girls $(\mathrm{O}=$ non-symptomatic, $\mathbf{0}$ = symptomatic). 
Table 1 Comparison of symptomatic and asymptomatic infants

\begin{tabular}{lll}
\hline & Symptomatic & Asymptomatic \\
\hline $\mathrm{n}$ & 13 & 47 \\
Birth weight (g) & $3540(2400-4830)$ & $3370(1800-4960)$ \\
Intrauterine growth retardation & $2(15)$ & $6(13)$ \\
Postnatal age (days) & $3(1-10)$ & $2(1-12)$ \\
Vaginal delivery (SVD) & $9(69)$ & $38(80)$ \\
Caucasian & $9(69)$ & $33(70)$ \\
Family history of atopy & $5(38)(2$ boys/3 girls) & $15(32)(6$ boys/9 girls) \\
Respiratory rate (breaths/minute) & $62(38-93)$ & $61(39-85)$ \\
TPTEF/TE & $0.349(0.298-0.442)$ & $0.412(0.303-0.520)$ \\
TGV (ml/kg) & $34(25-43)$ & $33(22-44)$ \\
Raw $\left(\mathrm{cm} \mathrm{H}_{2} \mathrm{O} / \mathrm{l} / \mathrm{s}\right)$ & $30(25-43)$ & $25(15-39)$ \\
sGaw $\left(\mathrm{cm} \mathrm{H} \mathrm{H}_{2} \mathrm{O} / \mathrm{s}\right)$ & $0.285(0.210-0.397)$ & $0.389(0.224-0.547)$ \\
\hline
\end{tabular}

Results are presented as median (range) or no. (\%).

$\mathrm{TPTEF} / \mathrm{TE}=$ time to reach peak tidal expiratory flow to total expiratory time; $\mathrm{TGV}=$ thoracic gas volume; Raw = airway resistance; $\mathrm{sGaw}=$ specific airway conductance.

Table 2 Prediction of persistence of respiratory symptoms at one year using a TPTEF/TE ratio of $<0.4$

\begin{tabular}{lrl}
\hline & Boys & Girls \\
\hline Sensitivity (\%) & 100 & 89 \\
Specificity (\%) & 62 & 65 \\
Positive predictive value (\%) & 33 & 47 \\
Negative predictive value (\%) & 100 & 94 \\
\hline
\end{tabular}

girls (0.398, range 0.303-0.520). Symptomatic boys and girls had lower TPTEF/TE ratios than non-symptomatic boys and girls, respectively ( $\mathrm{p}<0.02$ ). A TPTEF/TE ratio of less than 0.4 had a $92 \%$ sensitivity, $64 \%$ specificity, and $41 \%$ positive predictive value for respiratory symptoms in infancy. Similar values were obtained when boys and girls were considered separately (table 2 ).

\section{Discussion}

This study has shown that the TPTEF/TE ratio measured during the first week of life in infants who subsequently developed respiratory symptoms was lower than in those who remained asymptomatic. This difference was particularly apparent in boys. These results are compatible with earlier findings in infants examined at a mean age of two months, ${ }^{3}$ but the average TPTEF/TE of our study population was higher than that reported by Martinez et al. ${ }^{3}$ Carlsen et $a l,{ }^{89}$ however, have demonstrated a decrease in TPTEF/TE with increasing postnatal age during the first week of life. A review of the published data (table 3) shows that the trend persists over the first few months of life. The TPTEF/TE results we report are in keeping with both the findings of Carlsen et $a l^{8}$ and Stick et al. ${ }^{10}$ The latter group measured TPTEF/TE using a similar technique to that employed in the present study and, in addition, used respiratory inductance plethysmography (Respitrace).

The variability in measurements of TPTEF/TE was high, despite excluding $10 \%$ of traces as unsuitable for analysis. It has recently been suggested that at least 20 breaths (from two separate epochs) should be analysed to obtain a more representative value of TPTEF/ TE. ${ }^{11}$ Others have used a mean of 3-8 runs of five sequential breath $\mathrm{s}^{12}$ but analysis of 10 consecutive tidal breaths has been undertaken more commonly. ${ }^{31314}$ As a consequence, we chose the latter method. Our intraindividual variation is similar to that of Carlsen et al, ${ }^{8}$ but both results compare unfavourably with those of Stick et al. ${ }^{10}$ It seems unlikely, however, that this high variability is due to measurement technique as Stick quotes similar results using a face mask and pneumotachograph or a Respitrace. The high variability may be explained by the low postnatal age of our patients. ${ }^{11}$ Despite this, however, we were able to demonstrate significant differences between groups. Our study population containing 13 symptomatic infants, given the coefficient of variation of the measurement we noted, allowed us to detect a difference in TPTEF/TE of 0.066 between the groups with $80 \%$ power at the $5 \%$ level, which is below the difference reported in prospective studies. ${ }^{313}$ The positive predictive value of a low TPTEF/TE was relatively poor, however, but our symptomatic group consisted of only 13 infants and obstructive airway disease in early childhood is multifactorial so one factor alone could not be expected to have a high predictive value.

Infants who subsequently became symptomatic could not be identified by their birth weight, the proportion who were growth retarded, their postnatal age, mode of delivery, ethnic origin, or family history of atopy (table 1). As the numbers of symptomatic infants were small we did not attempt to subdivide the group according to the severity of their symptoms, so we cannot exclude that there may have been clinically important differences between these groups and the controls. The respiratory rate of the two groups did not differ significantly and was comparable to that reported by Carlsen et al. ${ }^{8}$ We found a $16 \%$ difference in the $\mathrm{TPTEF}_{\mathrm{P}} \mathrm{TE}_{\mathrm{E}}$ ratio between subsequently symptomatic and asymptomatic

Table 3 Previously published results of TPTEF/TE ratio

\begin{tabular}{|c|c|c|c|}
\hline Author & $T_{P T E F} / T_{E}$ & $\begin{array}{l}\text { Mean approximate } \\
\text { postnatal age of study group }\end{array}$ & $\begin{array}{l}\text { Mean respiratory } \\
\text { rate }(b p m)\end{array}$ \\
\hline Carlsen et al ${ }^{8}$ & 0.39 & One day & $58.9(25-114)$ \\
\hline Stick et al ${ }^{10}$ & $0.423-0.427$ & 58 hours $(33-114)$ & $47.9-46.9$ \\
\hline Stocks et al ${ }^{11}$ & 0.4 & Neonates ( $<2$ weeks $)$ & Not given \\
\hline Clarke et al ${ }^{12}$ & 0.38 & One month & 62 \\
\hline Clarke et al ${ }^{18}$ & 0.39 (boys) 0.41 (girls) & Neonates & Not given \\
\hline Stocks et al ${ }^{11}$ & 0.29 & Six weeks & Not given \\
\hline Young et al ${ }^{13}$ & $0.39(0.254$ in flow limited infants $)$ & One month & 42 \\
\hline Dezateux et al ${ }^{15}$ & 0.321 & 8 weeks & 46.5 \\
\hline Martinez et al ${ }_{14}^{3}$ & $0.312(0.254$ in wheezing infants $)$ & 8 weeks & Not given \\
\hline Martinez et al ${ }^{14}$ & 0.297 & 8 weeks & Not given \\
\hline Dezateux et al ${ }^{15}$ & 0.295 & 50 weeks & 30.5 \\
\hline Stocks et al & 0.27 & One year & Not given \\
\hline Clarke et al ${ }^{11}$ & 0.29 & One year & 29 \\
\hline
\end{tabular}

«Two techniques of measuring TPTEF/TE were employed. 
infants, which is similar to the $19 \%$ difference reported by Martinez et al. ${ }^{3}$ Clarke et al, ${ }^{12}$ however, found no significant differences between groups, but their population all had a parental history of atopy which may have masked differences.

In older children and adults the shape of the tidal breathing expiratory flow-time curve, quantified by calculation of TPTEF/TE, is closely correlated with specific airway conductance $(\mathrm{sGaw})^{2}$ - that is, the reciprocal of airway flow resistance divided by lung volume. A relationship between TPTEF/TE and sGaw has also been noted in infants older, but not younger, than three months by Dezateux et al ${ }^{15}$ who suggested that, in the younger infants, the pattern of expiratory flow may reflect dynamic maintenance of FRC as much as a response to airway calibre, hence the lack of a significant relationship between the TPTEF/TE ratio and $s$ Gaw in such patients. Interestingly, in this study we found the symptomatic infants to have significantly lower TPTEF/TE ratios and specific conductance. Low TPTEF/TE ratios have been found in patients with obstructive lung disease ${ }^{2}$ and bronchopulmonary dysplasia. ${ }^{16}$ Martinez et al found that boys with low TPTEF/TE values had a greatly increased risk of having a wheezing illness in the first year of life; ${ }^{3}$ in addition, those who wheezed during the first year of life and had at least one additional lower respiratory illness had lower initial levels of TPTEF/TE and conductance than "never" wheezers. ${ }^{14} \mathrm{It}$ was suggested that lower levels of airway conductance predispose, not to infection, but to clinically relevant airway obstruction following a suitable stimulus. ${ }^{14}$ Clarke et $a l^{12}$ recently failed to demonstrate a significant difference in tidal breathing parameters between infants who subsequently developed lower respiratory symptoms of cough or wheeze and those who did not. Their population, however, differed from that of the present or earlier study ${ }^{3}$ as only infants of atopic parents were included and this may have influenced the results. In both our study and that of Martinez et $a l^{3}$ a sex bias was found. Although this might suggest that boys have smaller airways relative to lung size than girls, ${ }^{17}$ we did not find a significant difference in the TPTEF/TE ratio between boys and girls but this is in contrast to an earlier report. ${ }^{8}$ With our study population of only 60 , however, a type II error cannot be excluded.

Our findings demonstrate the feasibility of measuring the TPTEF/TE ratio in the perinatal period in non-sedated infants. The positive predictive value of a low TPTEF/TE, however, was low and, if this result is confirmed in future studies, it undermines the usefulness of tidal breathing parameters as criteria for intervention strategies in an individual. Although a high variability in the measurement was noted, we were able to find statistically significant differences between infants who were subsequently symptomatic or asymptomatic and this technique may therefore be appropriate for large population studies.

Dr B Yuksel is supported by the Medical Research Council and Dr F Giffin by the Children Nationwide Medical Research Fund.

1 Yuksel B, Greenough A. Persistence of respiratory symptoms into the second year of life: predictive factors in infants born preterm. Acta Paediatr 1992;81:832-5.

2 Morris M, Lane D. Tidal expiratory flow patterns in airflow obstruction. Thorax 1981;36:135-42.

3 Martinez FD, Morgan WJ, Wright AL, Holdberg C, Taussig L, GHMA Pediatricians. Diminished lung function as a predisposing factor for wheezing respiratory illness in infants. $N$ Engl f Med 1988;319:1112-7.

4 DuBois AB, Botelho SY, Bedell GN, Marshall R, Comrie $\mathrm{JH}$. A rapid plethysmographic method for measuring thoracic gas volume: a comparison with a nitrogen washout method for measuring functional residual capacity in normal subjects. F Clin Invest 1956;35:322-6.

5 Dubois AB, Botelho SY, Comrie JH. A new method for measuring airway resistance in man using a body plethysmograph: values in normal subjects and in patients with respiratory disease. $\mathcal{f}$ Clin Invest 1956;35:327-32.

6 Yuksel B, Greenough A. Lung function in 6-20 month old infants born very preterm but without respiratory infants born very preterm but withou

7 Bland M, Altman D. Statistical methods for assessing agreement between two methods of clinical measurement. Lancet 1986;i:307-10.

8 Carlsen KCL, Magnus P, Carlsen KH. Lung function by tidal breathing in awake healthy newborn infants. Eur Respir F 1994;7:1660-8.

9 Carlsen KCL, Carlsen KH. Lung function in awake healthy infants in the first five days of life. Eur Respir $\mathcal{F}$ 1993;6:1496-500.

10 Stick SM, Ellis E, LeSouef PN, Sly PD. Validation of respiratory inductance plethysmography ("Respitrace") for the measurement of tidal breathing parameters in newborns. Pediatr Pulmonol 1992;14:187-91.

11 Stocks J, Dezateux CA, Jackson EA, Hoo A-F, Costeloe KL, Wade AM. Analysis of tidal breathing parameters in infancy: how variable is TPTEF/TE? Am $\mathcal{f}$ Respir Crit Car Med 1994;150:1347-54.

12 Clarke JR, Aston H, Silverman M. Evaluation of a tidal expiratory flow index in healthy and diseased infants. Pediatr Pulmonol 1994;1 17:285-90.

13 Young S, Arnott J, Le Souef PN, Landau LI. Flow limitation during tidal expiration in symptom free infants and the subsequent development of asthma. $\mathcal{F}$ Pediatr 1994 124:681-8.

14 Martinez FD, Morgan WJ, Wright AL, Holdber C, Taussig $\mathrm{LM}$. Initial airway function is a risk factor for recurren wheezing respiratory illness enduring the first three years of life. Am Rev Respir Dis 1991;143:312-6.

15 Dezateux CA, Stocks J, Dundas I, Jackson EA, Fletcher $M E$. The relationship between TPTEF/TE and specific airway conductance in infancy. Pediatr Pulmonol 1994; 18:299-307.

16 Morgan WJ, Tepper RS, Wilcox E, Taussig LM, GHMA Pediatricians. Shape and moment analysis of tidal expiration in normal (NL) and bronchopulmonary dysplasia infants (BPD). Am Rev Respir Dis 1984 129:A215.

17 Tepper RS, Morgan WJ, Cota K, Wright A, Taussig LM, GHMA Paediatricians. Physiological growth and development of the lung during the first year of life. Am Rev Respir Dis $1986 ; 134: 513-9$

18 Clarke J, Salmon B, Silverman M. Bronchial responsiveness in the neonatal period as a risk factor for wheezing in infancy. Am f Respir Crit Care Med 1995;151:1434-40. 